\title{
Rare Case Report of Multicentric Glioblastoma Multiforme Initially Mimicking With Herpes Simplex Encephalitis on MRI and Its Progression on Serial Imaging
}

\author{
Singh Rambir ${ }^{\mathrm{a}}$, Gupta Bharat ${ }^{\mathrm{b}, \mathrm{c}}$, Kardam Narendra ${ }^{\mathrm{b}}$, Gehlot Kushalbabu ${ }^{\mathrm{b}}$
}

\begin{abstract}
We report a case of a 58-year-old man with multicentric glioblastoma initially presenting as acute encephalitic illness. He presented with seizures and cognitive impairment in the emergency department of our hospital. Initially clinical and brain MRI findings were in favor of herpes simplex virus (HSV) encephalitis. The patient had initially improved after medical treatment. After 3 months, the patient complained of recurrent seizures. A follow-up brain MRI revealed marked increase in size of lesions and surrounding perilesional edema in the bilateral frontal lobes, cingulate gyrus and left insular cortex on T2weighted images and lesions showed enhancement on post-contrast T1-weighted images. Patient underwent right frontal craniotomy and resection of lesion in right frontal region. The treatment is surgery or palliative, and management is controversial. The prognosis, however, remains unfavorable. Therefore, in elderly patients presenting with multifocal lesions on MRI of brain or atypical encephalitic symptoms, the rare possibility of multicentric glioblastoma multiforme should be considered as differential diagnosis.
\end{abstract}

Keywords: Multicentric glioblastoma; HSV; Multiple intracranial lesions

\section{Introduction}

Multiple intracranial lesions represent a diagnostic dilemma and commonly represent metastases, lymphoma, infections, vascular or demyelinating disease. Multicentric gliomas are uncommon lesions but important. The rate of true multicentricity in gliomas is defined as lesions with no gross or microscopic linkage and has been reported to be $2-10 \%$ based on necropsy studies [1]. On CT scan, the incidence is reported to

Manuscript accepted for publication December 18, 2015

${ }^{a}$ MRI Center, RNT Medical College and MB Hospital, Udaipur, India ${ }^{b}$ Department of Radiodiagnosis, RNT Medical College, Udaipur, India ${ }^{\mathrm{c}}$ Corresponding Author: Gupta Bharat, Department of Radiodiagnosis, RNT Medical College, Udaipur, India. Email: bharatbalotra.bg@gmail.com

doi: http://dx.doi.org/10.14740/jmc2401w be $2.5 \%$ [2]. We discuss a case of multicentric glioblastoma multiforme (GBM) and review the relevant literature. Multiple gliomas were first observed by Virchow in 1864 and Bradley in 1880 [3-5] in their seminal paper published in 1962. The clinical presentation and imaging appearance of multicentric gliomas can mimic metastatic, encephalitis and other lesions. However, the treatment of these disease entities is considerably different [6]. Therefore, it becomes imperative to determine the histology of one of the lesions in the presence of multiple lesions on imaging [2].

\section{Case Report}

A 58-year-old man presented with general tonic clonic seizures and cognitive impairment in the emergency department of our hospital. Patient had mild fever and headache for 2 days. On neurological examination at the time of admission, memory impairment was observed, and no other focal neurological deficits were noted. There was no past history of medicine intake, drug abuse and toxins exposure. He had no familiar or personal history of psychiatric or neurological disorders, or in particular, of epileptic disease. He was a doctor and he had always done an efficient and competent job. He does not smoke and he drinks alcohol only occasionally.

Electroencephalogram was unremarkable which showed a bilateral, symmetrical $10 \mathrm{~Hz}$ alpha rhythm with normal arrest reaction to eyes opening, and did not reveal any epileptic rhythms or figures.

The routine electrocardiogram and blood investigations (blood cells count and morphometry, plasmatic glucose level, serum electrolytes, coagulation, kidney and liver function indexes) did not show any significant abnormalities. Blood pressure was normal. Examination of CSF revealed white blood cell of $7 / \mathrm{mm}^{3}\left(0-5 / \mathrm{mm}^{3}\right)$, protein level of $38 \mathrm{mg} / \mathrm{dL}$ (15 - 50 $\mathrm{mg} / \mathrm{dL}$ ) and glucose level of $71 \mathrm{mg} / \mathrm{dL}(45-80 \mathrm{mg} / \mathrm{dL})$. Herpes simplex virus-polymerase chain reaction (HSV-PCR) assay in the CSF was negative.

MRI study revealed asymmetrical multifocal lesions in bilateral frontal lobes, cingulate gyrus and left insular cortex with gyral swelling and effacement of sulci. The lesions appeared isointense on T1-weighted sequence (Fig. 1a) and hyperintense on T2-weighted (Fig. 1b) and FLAIR sequence (Fig. 1c, d). No obvious restriction was seen on the diffusion- 

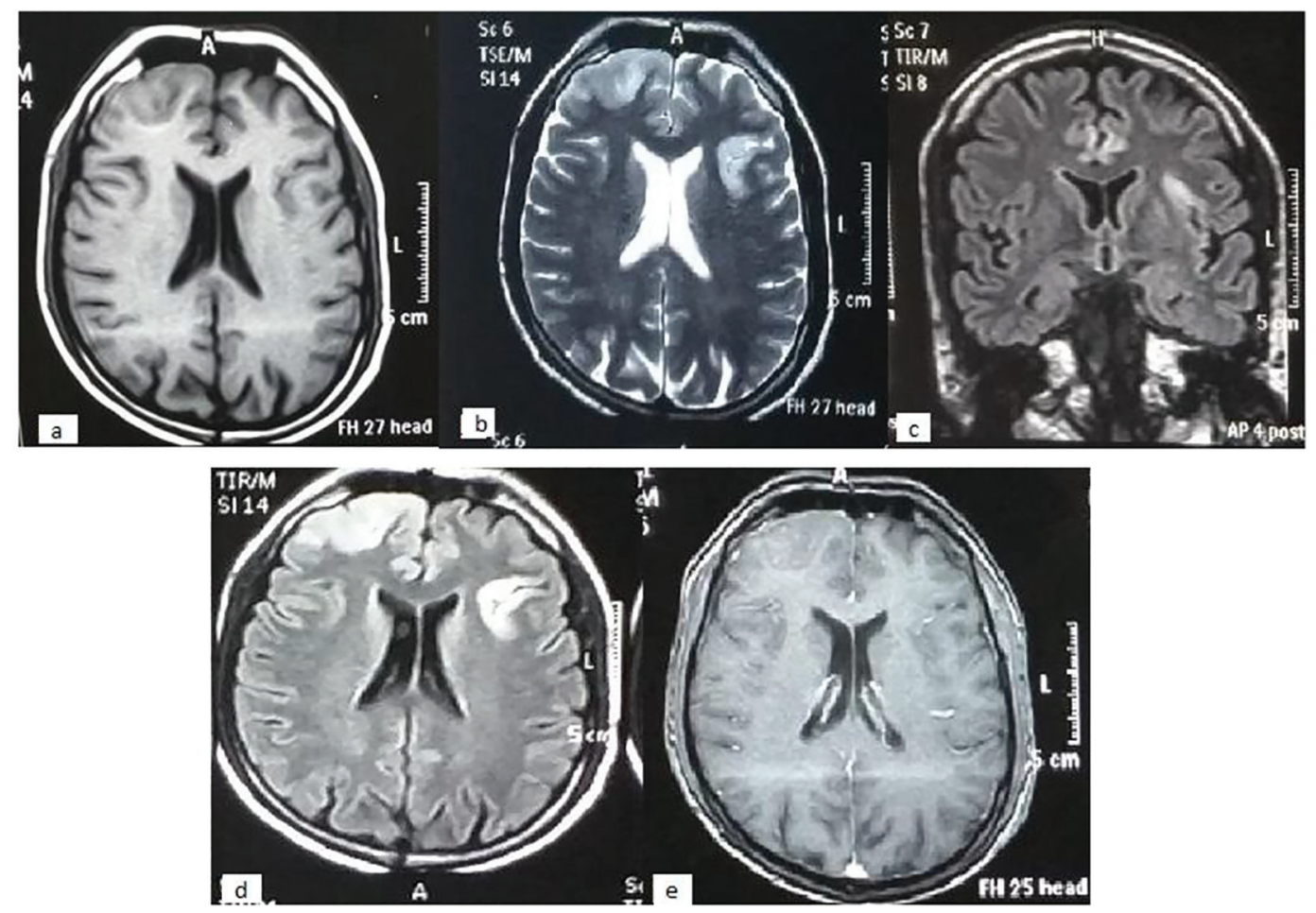

Figure 1. MR images of patient with multiple lesions in favor of encephalitis. (a) Axial image on T1-weighted sequence. (b) Axial image on T2-weighted sequence. (c, d) Coronal and axial image FLAIR sequence. (e) Axial image on T1-weighted post-contrast sequence.

weighted sequence and no appreciable contrast was seen on T1-weighted Gd sequence (Fig. 1e). On the basis of acute history and imaging features, possibilities of HSV encephalitis were considered. However, other possibilities of multifocal lesions like gliomatosis cerebri and transient postictal signal changes were also considered in the differential diagnosis. Close follow-up scan or stereotactic biopsy was advised. After treatment with intravenous acyclovir $(30 \mathrm{mg} / \mathrm{kg} /$ day for 14 days) and methylprednisolone ( $1 \mathrm{mg} /$ day for 5 days) under the diagnosis of herpes simplex encephalitis (HSE), his cognitive function was improved.

After 3 months, the patient had seizures and drowsy mentality. MRI of brain revealed multiple lesions at the same site and location of bilateral frontal lobes, cingulate gyrus and insular cortex, appearing iso- to hypointense on T1-weighted and hyperintense on T2-weighted (Fig. 2a) and FLAIR sequence. No obvious restriction was seen. There was enhancement of these lesions on T1-weighted Gd sequence (Fig. 2b, c). As imaging showed enhancement and increased size of lesions, there was suspicion of neoplastic lesion. Patient denied stereotactic

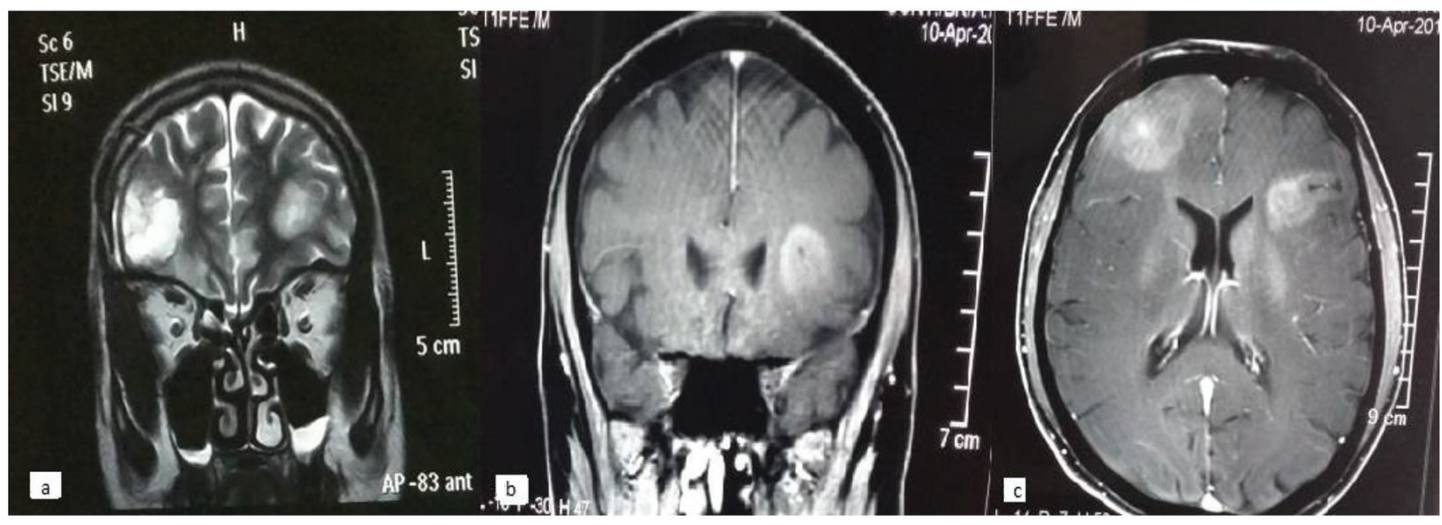

Figure 2. MRI after 3 months, showing multiple enhancing lesions arising suspicion of multicentric gliomas. (a) Axial image on T2-weighted sequence. (b) Coronal image on T1-weighted post-contrast sequence. (c) Axial image on T1-weighted post-contrast sequence. 

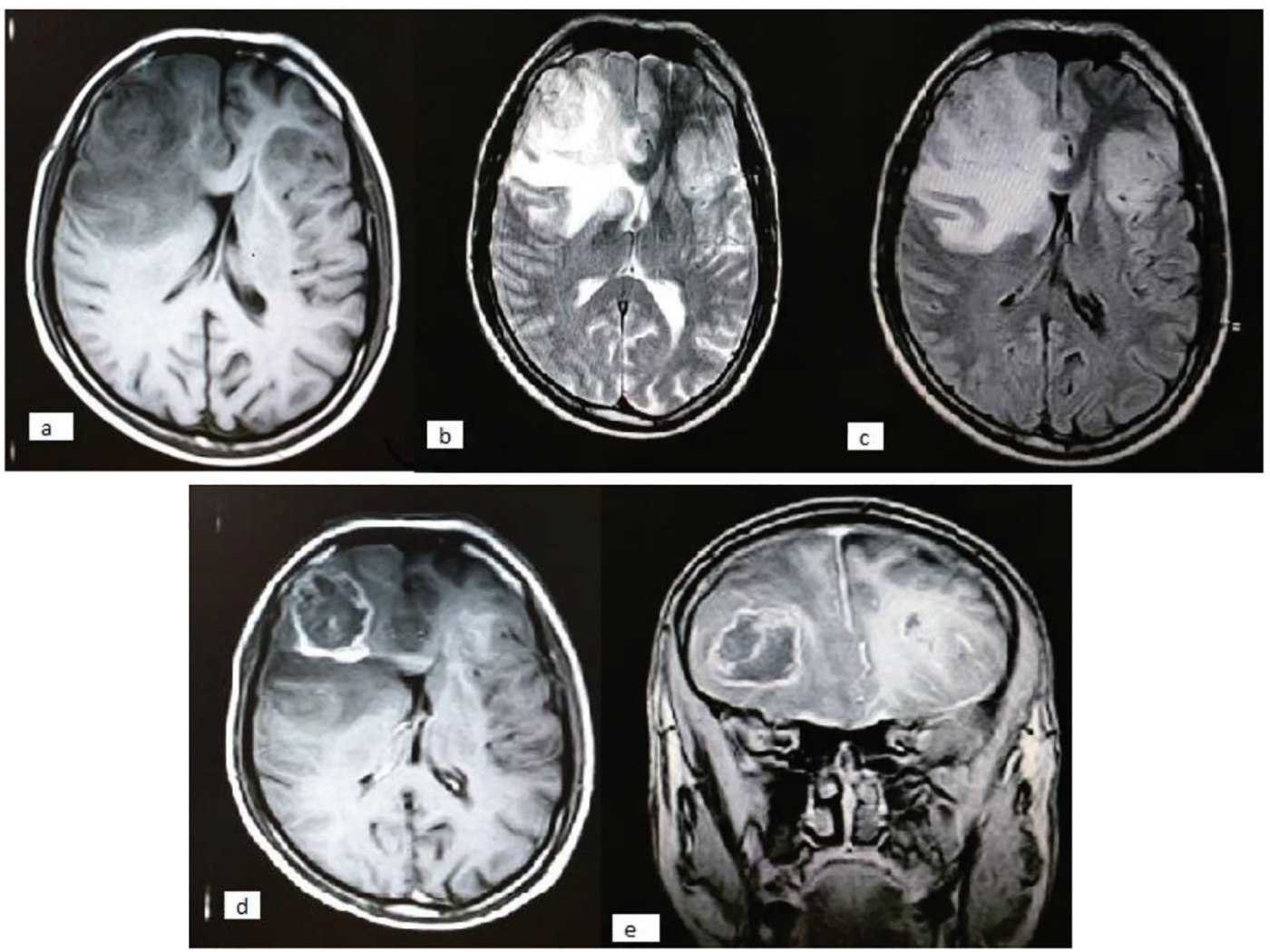

Figure 3. After 1 month of previous MRI, follow-up MRI suggesting of multicentric gliomas. (a) Axial image on T1-weighted sequence. (b) Axial image on T2-weighted sequence. (c) Axial image on FLAIR sequence. (d) Axial image on T1-weighted postcontrast sequence. (e) Coronal image on T1-weighted post-contrast sequence.

biopsy.

A follow-up scan was done after 1 month of previous imaging which showed multiple lesions at the same site appearing iso- to hypointense on T1-weighted (Fig. 3a) and hyperintense on T2 and FLAIR sequence (Fig. 3b, c). There was irregular peripheral enhancement on post-contrast study (Fig. $3 \mathrm{~d}$, e) with non-enhancing central necrotic/cystic areas. There was mass effect and midline shift towards left side. These images were suggesting of multicentric glioma and differential

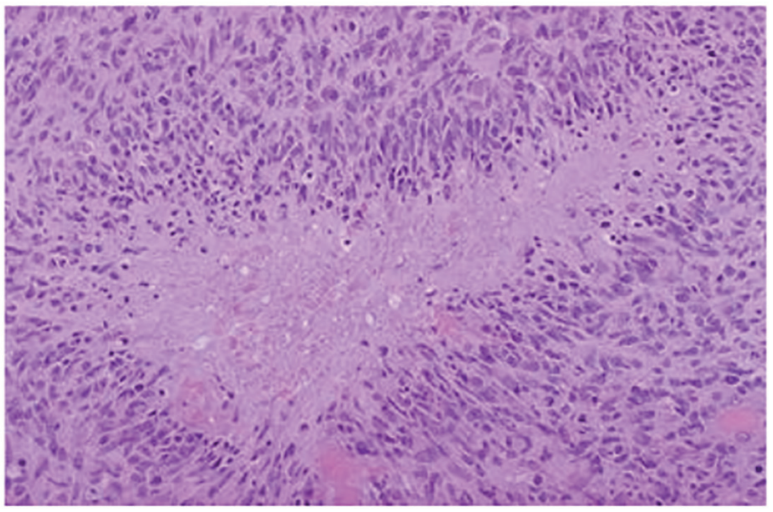

Figure 4. Histopathological section showing glioblastoma multiforme, WHO grade IV $(H \& E, \times 100)$. diagnosis included metastases.

Patient underwent right frontal craniotomy and resection of one of lesions in right frontal lobe was done. Histopathological examination of tissue showed gliofibrillary matrix and sheets of neoplastic astrocytes with moderate nuclear atypia and brisk mitosis. Multifocal microvascular proliferation and palisaded tumor necrosis were noted. Many gemistocytic tumor cells were seen, suggestive of GBM, WHO grade IV (Fig. 4).

Postoperative MRI showed changes in the form of craniotomy defect in right frontal region, dural enhancement and hemorrhage in right frontal lobe and associated with abnormal signals at peripheral part of resection cavity and cingulated gyrus appearing isointense on T1-weighted (Fig. 5a) and hyperintense on T2-weighted (Fig. 5b) and FLAIR sequences (Fig. 5c), suggesting of residual lesion. There was abnormal signal in the left frontal lobe, extending into insular cortex appearing isointense on T1-weighted hyperintense on T2weighted and FLAIR sequence. Lesion showed irregular peripheral enhancement on the post-contrast study with central cystic/necrotic area (Fig. 5d, e). Mild perilesional edema was seen. There was mass effect in form of effacement of the left lateral ventricle. Lesion in left frontal lobe increased in size as compared to preoperative MRI. The palliative IMRT was given at high dose on left frontal lobe, moderate dose at resection site and mild dose covering the entire brain sparing the 

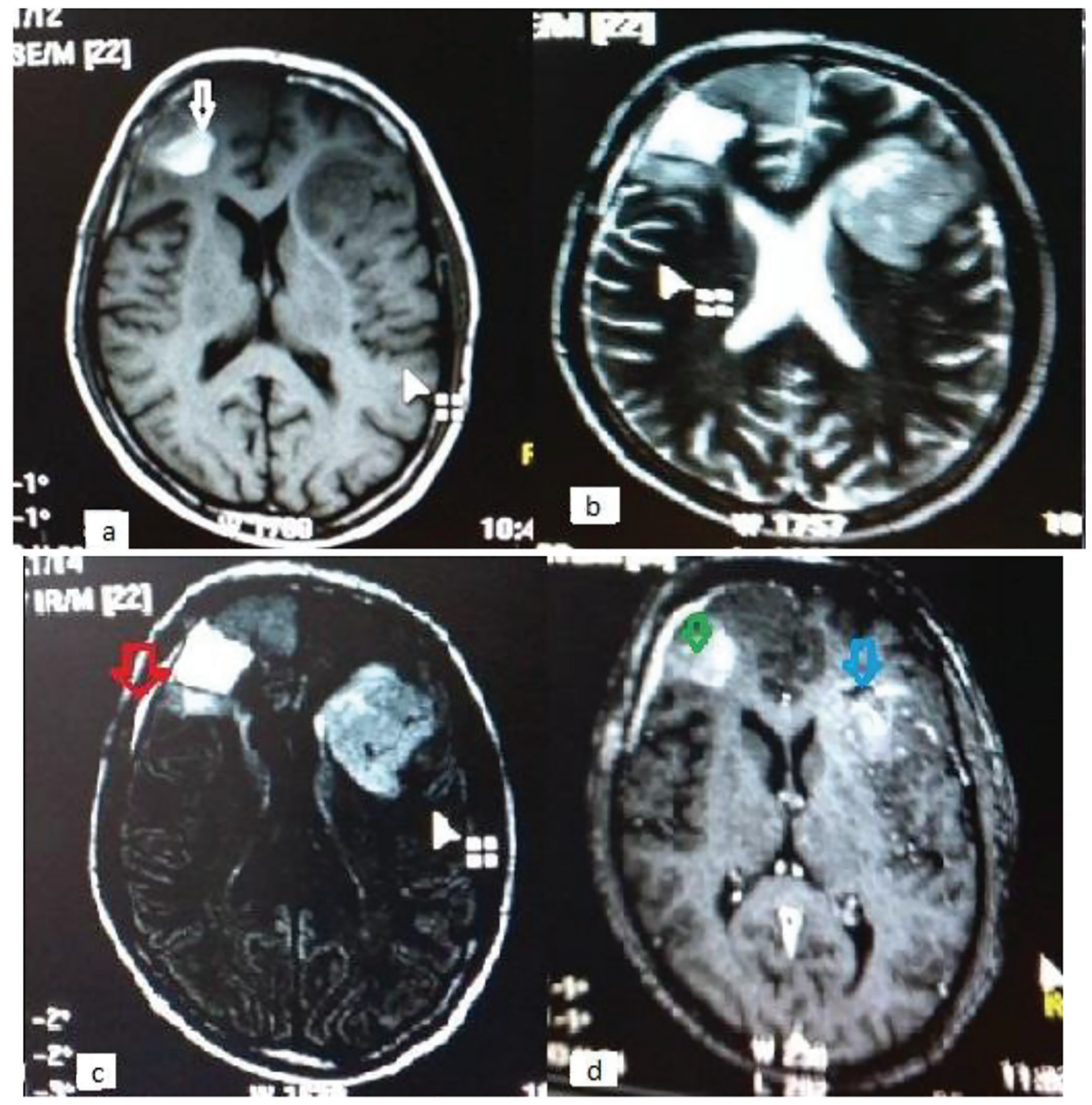

Figure 5. Postoperative MRI showing postoperative changes in the form of craniotomy defect (red arrow), hemorrhage (white arrow), residual lesion in right frontal lobe (green arrow) and lesion with internal necrosis in left frontal lobe (blue arrow) which increased in size compared to preoperative MRI. (a) Axial image on T1-weighted sequence. (b) Axial image on T2-weighted sequence. (c) Axial image on FLAIR sequence. (d) Axial image on T1-weighted post-contrast sequence.

vital structures like optic nerve, optic chiasma and pituitary gland. Patient was on follow-up.

\section{Discussion}

This case highlights unusual imaging features of multicentric glioma on MRI simulating with HSV encephalitis. However, similar clinicoradiological findings for glioblastoma and HSE can lead to delays in the diagnosis of glioblastoma and treatment of patients, and thus resulting in significant cerebral morbidity and poor prognosis.

HSE is the major cause of serious sporadic encephalitis with a predilection for the limbic system [4]. Therefore, intravenous acyclovir should be continued until the limbic system lesion is diagnosed as any other diseases except HSE, because failure to consider the possibility of HSE can lead to a delay in accurate diagnosis and proper treatment, with a significant risk of mortality and morbidity [1]. The HSV-PCR assay of the CSF is an invaluable test in the diagnosis of a patient with suspected HSE. However, it sometimes tends to remain falsenegative in the acute phase of HSE because the intensity of the PCR product band of an earlier CSF sample is weak [7]. For this reason, the negative result of the HSV-PCR assay in our case was overlooked and acyclovir continued to be intravenously administrated. The patient improved at the time of discharge. In retrospect, it may have been due to the corticosteroid effect but not acyclovir administration.

Primary and metastatic brain tumors, including glioblastoma, can present as acute encephalitis or encephalopathy, although the frequency is very low. Jasen [8] reported that one $(1.5 \%)$ out of 65 patients with acute encephalitis actually had a 
brain tumor (oligodendroglioma). Chen et al [9] reported that five $(5.3 \%)$ of 95 patients who were biopsy-negative for HSE had brain tumors, of whom three had primary CNS tumors (two patients with glioblastomas and one patient with primary CNS lymphoma) and two had metastatic colon adenocarcinoma. Further, Kimberlin [10] reported three patients with high grade gliomas mimicking acute viral encephalitis. They suggested that stereotactic brain biopsy should be considered in patients with temporal lobe masses if a definitive diagnosis using PCR assays for common viruses is unavailable. If we had performed stereotactic brain biopsy on our patient based on the initial CNS manifestations, functional deficits would have been much less severe.

First, MRI is more sensitive than CT in the detection of brain tumors. However, patients whose initial MRIs were negative or had mild abnormalities, and soon thereafter had high grade glioma, were occasionally encountered. After all, in suspected tumors, serial brain imaging studies are needed to document the evolution of brain tumor and to rule out underlying encephalitis.

The imaging appearance of multifocal and multicentric GBM is indistinct from that of metastases, with MRI displaying multiple contrast-enhancing masses $[9,11]$. Certain MRI features such as variable lesion morphology, mild peritumoral edema, and irregular tumor margins can suggest the diagnosis of multifocal or multicentric GBM [12-14]. Since metastasis from extracranial primary tumors is the most common diagnosis associated with multiple brain masses, histopathological verification is imperative before making a diagnosis of metastasis, especially in patients with no known primary neoplasm $[3,11]$. The diagnostic workup for multifocal and multicentric GBM is generally the same as that for solitary GBM. Dissemination of GBM, however, can occur intracranially or throughout the spinal axis [15]. In such cases of GBM with leptomeningeal gliomatosis, either suspected clinically or radiographically due to the presence of leptomeningeal enhancement or hydrocephalus, complete neuroaxis MRI should be obtained. Cytological examination of the CSF can be used to confirm the diagnosis. Batzdorf and Malamud characterized the modes of growth in gliomas by establishing criteria to distinguish multiple and multicentric gliomas [16]. Others have attempted additional classifications based on pathologic and radiologic criteria [4, 17]. Multifocal replaces multiple in the modern day classification. Therefore, multifocal glioma consists of tumors separated by white matter tracts within the same hemisphere, whereas multicentric glioma consists of tumors in opposite hemispheres or separated by the tentorium.

Multifocal and multicentric GBMs do not exhibit any histopathologic characteristics that differentiate them from typical, solitary supratentorial GBM [16, 18]. Pathognomonic features of GBM include pseudopalisading necrosis and neovascularization [19]. The individual tumors in cases of multicentric GBM usually have the same pathologic appearance $[16,20]$. Although there is still no unified theory regarding the pathogenesis of multifocal and multicentric GBM, several hypotheses have been developed. According to earlier theories, multicentricity arises from two events $[8,10]$. The first stage is neoplastic transformation, in which a wide field becomes more susceptible to neoplastic growth. The second stage is tu- mor proliferation at two or more activated sites that can occur simultaneously in response to various stimuli including biochemical, hormonal, and viral triggers. More contemporary theories have looked at molecular associations. For example, there is a reported association between $p 53$ mutations and multifocal GBM that correlates the pattern of $p 53$ mutation to tumor migration and augmented growth. In a study of the growth factor receptor c-Met in GBM, one group found that $42.9 \%$ of tumors that overexpressed c-Met displayed invasive and multifocal features on initial MRI, whereas only $17.1 \%$ of tumors with little or no c-Met expression had similar characteristics $(\mathrm{P}$ $=0.036$ ) [21]. This molecular and genetic characterization of multifocal GBM has thereby implicated particular oncogenes and growth factors in the pathogenesis of multifocal and multicentric GBM.

In addition to the molecular pathways involved in multifocality and multicentricity, studies have correlated the tumor pattern at diagnosis and recurrence with the spatial relationship to the subventricular zone (SVZ) and cortex as seen on MRI [4]. More specifically, patients with a contrast-enhancing lesion contacting the SVZ and infiltrating the cortex were most likely to have multifocal disease at the time of diagnosis and distant tumor recurrence. On the other hand, patients with a contrast-enhancing lesion neither contacting the SVZ nor infiltrating the cortex always had solitary lesions and contiguous tumor recurrences. Neural stem cells within the SVZ may give rise to multiple and multicentric GBM. Neural stem cells have been found to express matrix metalloproteinases, which are proteolytic enzymes implicated in tumor spread [4]. Furthermore, the SVZ is thought to be a highly permissive environment for tumor growth and cellular migration.

Currently, there are no clear guidelines regarding the optimal management of multifocal and multicentric GBM [12]. While the extent of resection is established as an independent determinant of survival in patients with solitary GBM, the role of surgery for multifocal and multicentric GBM remains controversial $[8,12,22]$. Two strategies have been described. First one is surgery done in our patient, aggressive resection of one tumor focus, biopsy alone followed by chemotherapy and radiation treatment, and second one is multiple craniotomies during a single operation with no clear indication of which modality is superior [12, 23-25]. The standard radiation treatment for GBM includes conformal radiotherapy that encompasses the tumor volume and margin along with concurrent and adjuvant temozolomide $[2,25,26]$. Regarding radiotherapy for multifocal and multicentric GBM, a study found no significant difference in the median time to progression or median survival time between conformal radiotherapy and whole brain radiation treatment [25].

Additionally, the role of radiotherapy to control infratentorial GBM is not yet defined [7, 27, 28]. Some studies support the use of craniospinal radiotherapy with posterior fossa boost for malignant cerebellar gliomas, especially in children [27]. However, other studies have found no benefit with craniospinal radiotherapy in GBM in adults and support whole brain radiation treatment with or without posterior fossa boost [7]. Most authors agree that craniospinal irradiation is reasonable if CSF dissemination occurs [28]. In patients with leptomeningeal gliomatosis, other treatments could also include intrath- 
ecal chemotherapy and CSF shunting for associated hydrocephalus.

\section{Conclusion}

Multicentric glioblastoma is a rare entity. The clinical presentation may develop extremely fast and can be characterized quite exclusively by cognitive symptoms; such symptoms can be very difficult to place, especially when focal neurological signs are not present. It is rare that high-grade brain tumors may present as acute encephalopathy with limbic system involvement by brain imaging.

In this particular case, the initial imaging was mimicking with acute HSV encephalitis and we could not expect a so relevant evolution in such short time. Therefore, an intensive follow-up with short repeat brain imaging as well as stereotactic biopsy is necessary in such patients if definite evidence for the diagnosis of HSE can not be obtained.

\section{Acknowledgement}

We would like to express our special thanks of gratitude to Dr. Sunil Gupta for his constant encouragement without which this assignment would not have been possible. We also take this opportunity to express a deep sense of gratitude to our friends Dr. Manisha Singhal, Dr. Shruti Agarwal and Dr. Sagar Satpute for their cordial support, valuable information and guidance, which helped us in completing this task through various stages.

\section{Competing Interests}

The authors declare that they have no competing interests.

\section{References}

1. Bussone G, Sinatra MG, Boiardi A, Lazzaroni M, Mariani C, Allegranza A. A case of glioblastoma with multiple centers above and below the tentorium. J Neurol. 1979;221(3):187-192.

2. Van Tassel P, Lee YY, Bruner JM. Synchronous and metachronous malignant gliomas: CT findings. AJNR Am J Neuroradiol. 1988;9(4):725-732.

3. Bradley WL. Case of glio-sarcomatous tumors of the cerebrum and cerebellum. Proceedings of the Connecticut Medical Society. 1880;2:39-41.

4. Salvati M, Oppido PA, Artizzu S, Fiorenza F, Puzzilli F, Orlando ER. Multicentric gliomas. Report of seven cases. Tumori. 1991;77(6):518-522.

5. Takeda F, Tanaka S, Kawabuchi J, Nakajima T. [Multicentric gliomas: report of three autopsied cases and a review of the criteria of multicentricity (author's transl)]. Neurol Med Chir (Tokyo). 1976;16(3 pt 2):207-214.

6. Rao KC, Levine H, Itani A, Sajor E, Robinson W. CT findings in multicentric glioblastoma: diagnostic-pathologic correlation. J Comput Tomogr. 1980;4(3):187-192.

7. Chadduck WM, Roycroft D, Brown MW. Multicentric glioma as a cause of multiple cerebral lesions. Neurosurgery. 1983;13(2):170-175.

8. Jansen EP, Dewit LG, van Herk M, Bartelink H. Target volumes in radiotherapy for high-grade malignant glioma of the brain. Radiother Oncol. 2000;56(2):151-156.

9. Chen W. Clinical applications of PET in brain tumors. J Nucl Med. 2007;48(9):1468-1481.

10. Kimberlin DW. Management of HSV encephalitis in adults and neonates: diagnosis, prognosis and treatment. Herpes. 2007;14(1):11-16.

11. Kudo H, Tanaka M, Urui S, Suzuki H, Tamaki N, Matsumoto S. Multicentric glioblastoma multiforme occurring in the supra- and the infratentorial regions--case report. Neurol Med Chir (Tokyo). 1990;30(5):334-338.

12. Whitley RJ, Cobbs CG, Alford CA, Jr., Soong SJ, Hirsch MS, Connor JD, Corey L, et al. Diseases that mimic herpes simplex encephalitis. Diagnosis, presentation, and outcome. NIAD Collaborative Antiviral Study Group. JAMA. 1989;262(2):234-239.

13. Willis RA. Pathology of the Tumors. Butterworth: London, UK. 4th edition. 1967.

14. Hassaneen W, Levine NB, Suki D, Salaskar AL, de Moura Lima A, McCutcheon IE, Prabhu SS, et al. Multiple craniotomies in the management of multifocal and multicentric glioblastoma. Clinical article. J Neurosurg. 2011;114(3):576-584.

15. Osborn AG. Diagnostic Neuroradiology. Mosby, St. Louis, Mo, USA, 1994.

16. Batzdorf U, Malamud N. The Problem of Multicentric Gliomas. J Neurosurg. 1963;20:122-136.

17. Budka H, Podreka I, Reisner T, Zeiler K. Diagnostic and pathomorphological aspects of glioma multiplicity. Neurosurg Rev. 1980;3(4):233-241.

18. Kuroiwa T, Numaguchi Y, Rothman MI, Zoarski GH, Morikawa M, Zagardo MT, Kristt DA. Posterior fossa glioblastoma multiforme: MR findings. AJNR Am J Neuroradiol. 1995;16(3):583-589.

19. Lafitte F, Morel-Precetti S, Martin-Duverneuil N, Guermazi A, Brunet E, Heran F, Chiras J. Multiple glioblastomas: CT and MR features. Eur Radiol. 2001;11(1):131-136.

20. Parsa AT, Wachhorst S, Lamborn KR, Prados MD, McDermott MW, Berger MS, Chang SM. Prognostic significance of intracranial dissemination of glioblastoma multiforme in adults. J Neurosurg. 2005;102(4):622-628.

21. Misra BK, Steers AJ, Miller JD, Gordon A. Multicentric glioma presenting with hemorrhage. Surg Neurol. 1988;29(1):73-76.

22. Krex D, Mohr B, Appelt H, Schackert HK, Schackert G. Genetic analysis of a multifocal glioblastoma multiforme: a suitable tool to gain new aspects in glioma development. Neurosurgery. 2003;53(6):1377-1384; discussion 1384.

23. Kyritsis AP, Bondy ML, Xiao M, Berman EL, Cunningham JE, Lee PS, Levin VA, et al. Germline p53 gene mutations in subsets of glioma patients. J Natl Cancer Inst. $1994 ; 86(5): 344-349$. 
24. Kong DS, Song SY, Kim DH, Joo KM, Yoo JS, Koh JS, Dong SM, et al. Prognostic significance of c-Met expression in glioblastomas. Cancer. 2009;115(1):140-148.

25. Lim DA, Cha S, Mayo MC, Chen MH, Keles E, VandenBerg S, Berger MS. Relationship of glioblastoma multiforme to neural stem cell regions predicts invasive and multifocal tumor phenotype. Neuro Oncol. 2007;9(4):424429.

26. McGirt MJ, Chaichana KL, Gathinji M, Attenello FJ, Than $\mathrm{K}$, Olivi A, Weingart JD, et al. Independent association of extent of resection with survival in patients with malignant brain astrocytoma. J Neurosurg. 2009;110(1):156-162.

27. Salvati M, Cervoni L, Celli P, Caruso R, Gagliardi FM. Multicentric and multifocal primary cerebral tumours. Methods of diagnosis and treatment. Ital J Neurol Sci. 1997;18(1):17-20.

28. Showalter TN, Andrel J, Andrews DW, Curran WJ, Jr., Daskalakis C, Werner-Wasik M. Multifocal glioblastoma multiforme: prognostic factors and patterns of progression. Int J Radiat Oncol Biol Phys. 2007;69(3):820-824. 